\title{
The cyanobacterium Synechococcus sp. strain PCC 7942 contains a second alkaline phosphatase encoded by phoV
}

\author{
Klaus-Uwe Wagner, ${ }^{1}$ Bernd Masepohl ${ }^{2} \dagger$ and Elfriede K. Pistorius ${ }^{1}$
}

Biologie VIII:

Zellphysiologie1, and Biologie VI: Genetik? Universităt Bielefeld, 33501 Bielefeld, FRG
Author for correspondence: Elfriede K. Pistorius. Tel: +49521 106 5601. Fax: +49 5211065626.

\begin{abstract}
A gene (phoV) encoding an alkaline phosphatase from Synechococcus sp. strain PCC 7942 was isolated by screening a plasmid gene bank for expression of alkaline phosphatase activity in Escherichia coli JM103. Two independent clones carrying the same alkaline-phosphatase-encoding gene were isolated. One of these clones (pKW1) was further analysed and the nucleotide sequence of a contiguous 3234 bp DNA fragment was determined. Two complete open reading frames (ORF1 and pho $V$ and an incomplete ORF3 were identified reading in the same direction. The deduced phoV gene product showed $34 \%$ identity to the alkaline phosphatase PhoA from Zymomonas mobilis, and the $\mathrm{N}$-terminal part of the putative ORF3 protein exhibited $57 \%$ identity to a protein of unknown function from Frankia sp. Insertional inactivation of the Synechococcus PCC 7942 phoV gene failed, indicating an essential role for either the phoV or the ORF3 gene product. PhoV consists of 550 amino acid residues, resulting in a molecular mass of $61.3 \mathrm{kDa}$. To overexpress the Synechococcus PCC 7942 phoV gene in E. coli, plasmid pKW1 was transformed into a phoA mutant of E. coli (CC118). In E. coli strain CC118(pKW1) PhoV was expressed constitutively with high rates of activity, and was shown to be membrane associated in the periplasmic space. After partial purification of the recombinant PhoV, it was shown that, like other alkaline phosphatases, the Synechococcus PhoV had a broad pH optimum in the alkaline region and a broad substrate specificity for phosphomonoesters, required $\mathrm{Zn}^{2+}$ for activity, and was inhibited by phosphate. In contrast to several other alkaline phosphatases, PhoV was inhibited by $\mathrm{Mn}^{2+}$. Due to the lack of a Synechococcus PCC 7942 phoV mutant strain, the function of PhoV remains uncertain. However, the present results show that Synechococcus PCC 7942 has a second, probably phosphate-irrepressible, alkaline phosphatase (PhoV, 61.3 kDa) in addition to the phosphate-repressible enzyme (PhoA, $145 \mathrm{kDa}$ ) already described.
\end{abstract}

Keywords: alkaline phosphatase, Synechococcus PCC 7942, cyanobacteria, phoV gene

\section{INTRODUCTION}

Cyanobacteria and other micro-organisms constantly monitor environmental conditions and adjust their struc-

† Present address: Botanisches Institut der Universităt Bonn, 53115 Bonn, FRG.

Abbreviations: APase, alkaline phosphatase; BCIP, 5-bromo-4-chloro3-indolyl phosphate; NBT, nitroblue tetrazolium; 4-NP, 4-nitrophenol; 4-NPP, 4-nitrophenyl phosphate.

The GenBank accession number for the sequence reported in this paper is 248801 . ture and physiology accordingly. Phosphate is a nutrient required at high level for cell growth and is frequently the limiting nutrient, since it often exists in forms not readily available. To cope with phosphate-limiting conditions, cells store polyphosphate reserves which allow them to withstand short periods of phosphate deprivation, and express extracellular phosphatases to obtain phosphate from organic substrates which are present in the surrounding medium, but which cannot penetrate the cell membrane (Carr \& Mann, 1994; Grossman et al., 1994). When growing in a phosphate-deficient environment, a number of micro-organisms induce the synthesis of 
alkaline phosphatase (APase). Most of these APases are located in the periplasmic space as soluble enzymes (Cheng \& Costerton, 1973; Bhatti et al., 1976) but membraneassociated enzymes have also been found (Von Tigerstrom \& Stelmaschuk, 1986; Baoudene-Assali et al., 1993). The most thoroughly investigated prokaryotic APase is that of Escherichia coli (Wanner, 1987; Wyckoff, 1987).

APase activity varies with phosphate composition in Anabaena flos-aquae (Bone, 1971; Healy, 1973), Synechococcus sp. strain PCC 7942 (Ihlenfeldt \& Gibson, 1975; Block \& Grossman, 1988), and Plectonema boryanum (Doonan \& Jensen, 1977). Ultrastructural localization of APase in $P$. boryanum showed that the enzyme was present in layer 3 (periplasmic space) of the cell wall. Extensive studies have been carried out on a derepressible APase from Synechococcus PCC 7942 (Block \& Grossman, 1988; Ray et al., 1991). This atypical APase has a subunit molecular mass of $145 \mathrm{kDa}$ and is located in the periplasmic space in association with the cell wall or cytoplasmic membrane. Insertional inactivation of the corresponding pho $A$ gene resulted in loss of extracellular, phosphate-regulated phosphatase activity, but did not alter the capacity of the cells for phosphate uptake, nor their viability when maintained on phosphate-sufficient or -deficient medium. The mutant showed slightly greater overall external APase activity than the wild-type, but there was no further increase in APase activity after transfer of the mutant cells to phosphate-free medium. These results imply that Synechococcus PCC 7942 cells have at least one additional APase as well as the atypical $145 \mathrm{kDa} \mathrm{PhoA}$ (Ray et al., 1991). This additional APase is not repressed by phosphate, in contrast to the phosphate-repressible PhoA. Here we report the identification and sequence analysis of a second gene ( $p h o V$ ) encoding an APase in Synechococcus PCC 7942, and the partial characterization of the $p h o V$ gene product.

\section{METHODS}

Bacterial strains, plasmids and growth conditions. Synechococcus PCC 7942 was obtained from the Pasteur Culture Collection of Cyanobacterial Strains, Paris, France. The E. coli JM103 host for the plasmids pUC19, pSVB28 and derivatives was the same as described by Messing et al. (1981), and the APase-free $E$. coli strain CC118 was the same as described by Manoil \& Beckwith (1985). Synechococcus PCC 7942 was grown in BG11 medium (Rippka, 1988) as previously described (Engels et al., 1992). E. coli strains were cultivated at $37^{\circ} \mathrm{C}$ in LB medium (Miller, 1972) or on PA plates $\left[1 \mathrm{l}\right.$ distilled $\mathrm{H}_{2} \mathrm{O}$ contained $17.5 \mathrm{~g}$ antibiotic medium no. 3 (assay broth, Oxoid) and $16.0 \mathrm{~g}$ bacteriological agar (Oxoid)]. Antibiotics were added at the following concentrations: ampicillin, $150 \mathrm{mg} \mathrm{l}^{-1}$ for E. coli and $0.5 \mathrm{mg} \mathrm{l}^{-1}$ for Synechococcus; chloramphenicol, $50 \mathrm{mg} \mathrm{l}^{-1}$ for $E$. coli and $7.5 \mathrm{mg} \mathrm{l}^{-1}$ for Synechococcus. To achieve phosphate deprivation, cells were harvested by centrifugation at $5000 \mathrm{~g}$ for $15 \mathrm{~min}$, washed with $\mathrm{LB}$ medium in which $\mathrm{KH}_{2} \mathrm{PO}_{4}$ was replaced by $\mathrm{KCl}$ (phosphate-free medium) and then resuspended in the same medium at about a threefold lower cell density.

The plasmids used in this study were pUC19 (Yanisch-Perron $e t$ al., 1985), pSVB28 (Arnold \& Pühler, 1988) and pRB5, a pUC19 derivative carrying the Synecbococcus PCC 6301 psbO gene inactivated by a Cm${ }^{\mathrm{r}}$ cassette (Bockholt $e$ t al., 1991). Details of the pUC19 and pSVB28 derivatives constructed in this paper are given in the legend to Fig. 1.

Cloning and hybridization procedures. Isolation of total DNA from Synecbococcus PCC 7942 and of plasmid DNA from E. coli, cloning procedures and restriction analysis were performed by standard methods (Sambrook et al., 1989). Hybridization experiments were carried out using the DIG labelling and detection kit (Boehringer Mannheim).

To isolate the APase-encoding gene ( $p$ ho $\mathrm{V}$ ) of Synecbococcus PCC 7942 , a size-fractionated genomic library containing $2-10 \mathrm{~kb}$ DNA fragments (from a partial Sau3AI digestion), cloned into the Bam HI site of vector plasmid pUC19, was transformed into E. coli JM103. Ninety-two percent of the clones were shown to contain an insert. Colonies were transferred onto nitrocellulose membranes (Millipore HAHYO8250, filter type HA, pore size $0.45 \mu \mathrm{m})$ and were incubated under an atmosphere of chloroform vapour for $15 \mathrm{~min}$. Subsequently, the membranes were incubated in lysis buffer $(100 \mathrm{mM}$ Tris $/ \mathrm{HCl}, \mathrm{pH} 7 \cdot 8$, containing $150 \mathrm{mM} \mathrm{NaCl} 5 \mathrm{mM} \mathrm{MgCl}_{2}, 1 \cdot 5 \% \mathrm{BSA}, 1 \mu \mathrm{g} \mathrm{DNase} \mathrm{I} \mathrm{ml}^{-1}$ and $40 \mu \mathrm{g}$ lysozyme $\mathrm{ml}^{-1}$ ) for $15 \mathrm{~h}$ at room temperature. Membranes were washed twice in TNT buffer $(10 \mathrm{mM}$ Tris/ $\mathrm{HCl}, \mathrm{pH} 8.0$, containing $150 \mathrm{mM} \mathrm{NaCl}, 0 \cdot 05 \%$ Tween 20 ) for $30 \mathrm{~min}$ at room temperature. Remaining cell debris was wiped from the membranes before washing in colony-assay buffer $(100 \mathrm{mM}$ Tris/ $\mathrm{HCl}, \mathrm{pH} 7 \cdot 6$, containing $1 \mathrm{mM} \mathrm{ZnCl}$ and $0.1 \%$ Triton $\mathrm{X}-100$ ) for $10 \mathrm{~min}$ at room temperature. Each membrane preparation (about $50 \mathrm{~cm}^{2}$ ) was incubated in $10 \mathrm{ml}$ fresh colony-assay buffer before $50 \mu \mathrm{l} \mathrm{NBT}\left(50 \mathrm{mg} \mathrm{ml}^{-1}\right.$ in $70 \%$ dimethylformamide) and $25 \mu \mathrm{l} \mathrm{BCIP}\left(50 \mathrm{mg} \mathrm{ml}^{-1}\right.$ in $100 \%$ dimethylformamide) were added. After 5-15 min incubation at room temperature, clones containing the APase-encoding gene (phoV) from Synechococcus PCC 7942 were identified by their red colour.

DNA sequencing. Appropriate restriction fragments were cloned into the pSVB28 sequencing vector (Arnold \& Pühler, 1988), resulting in hybrid plasmids pKW13 and pKW14, and nested deletions were introduced with the exonuclease III deletion kit (Pharmacia). Sequencing of both strands was performed using the Automatic Laser Fluorescent DNA Sequencer (Pharmacia). DNA sequences were analysed using the Staden software package (Staden, 1986) and PC/GENE (IntelliGenetics). Homology searches were performed using the Fasta and tFasta algorithms (Pearson \& Lipman, 1988).

Biochemical methods. For APase measurements, either whole E. coli cell suspensions or cell extracts were used. Cells were harvested by centrifugation, washed once with APase buffer $\left(0 \cdot 1 \mathrm{M}\right.$ Tris/ $\mathrm{HCl}, \mathrm{pH} 7 \cdot 6$, containing $1 \mathrm{mM} \mathrm{ZnCl} \mathrm{Zn}_{2}$ and $1 \mathrm{mM}$ $\mathrm{MgCl}_{2}$ ) and resuspended in the same buffer. Cell extracts were obtained by passing cell suspensions through a French press at $138 \mathrm{MPa}$. The osmotic shock procedure for $E$. coli cells was performed according to Neu \& Heppel (1965). Protein determinations were performed according to Bradford (1976), and SDS-PAGE was done as previously described (Engels et al., 1992).

APase actvity was determined in a reaction mixture of $2.5 \mathrm{ml}$ containing: $100 \mathrm{mM}$ Tris/ $\mathrm{HCl}, \mathrm{pH} 7.6,1 \mathrm{mM} \mathrm{ZnCl}, 1 \mathrm{mM}$ $\mathrm{MgCl}_{2}, 2 \%$ (v/v) Triton X-100, $10 \mathrm{mM}$ 4-nitrophenyl phosphate (4-NPP), and the sample. After incubation for $5 \mathrm{~min}$ at $37^{\circ} \mathrm{C}$ the reaction was stopped by adding $0.5 \mathrm{ml} 1 \mathrm{M} \mathrm{NaOH}$. After centrifugation at $5000 \mathrm{~g}$ for $15 \mathrm{~min}$, the absorbance of the supernatant was measured at $405 \mathrm{~nm}$ and compared to a standard absorbance curve of 4-nitrophenol (Sigma).

Partial purification of PhoV. E. coli CC118(pKW1) cells were harvested by centrifugation and washed once with $10 \mathrm{mM}$ 
Tris/ $\mathrm{HCl}$, pH $7 \cdot 6$, containing $1 \mathrm{mM}$ EDTA, and then resuspended in $10 \mathrm{mM}$ Tris $/ \mathrm{HCl}, \mathrm{pH} 7 \cdot 6$, containing $1 \mathrm{mM}$ $\mathrm{MgCl}_{2}$ and $1 \mathrm{mM} \mathrm{ZnCl}$ (buffer A). After breaking the cells by French press treatment at $138 \mathrm{MPa}$ and centrifugation of the extract at $30000 \mathrm{~g}$ for $35 \mathrm{~min}$, the pellet was washed three times with buffer A. Subsequently, PhoV was extracted from the pellet by washing with $1 \mathrm{M}$ Tris $/ \mathrm{HCl}, \mathrm{pH} 7.6$, containing $10 \mathrm{mM} \mathrm{MgCl}_{2}, 10 \mathrm{mM} \mathrm{ZnCl}_{2}$, and $2 \%$ Triton X-100 (buffer B). After centrifugation at $30000 \mathrm{~g}$ for $35 \mathrm{~min}$, the suspension was dialysed against buffer $A$. This resulted in precipitation of the major part of the PhoV. After centrifugation, the pellet fraction containing the PhoV was washed with buffer A containing $2 \%$ Triton X-100 to extract proteins which become solubilized by Triton X-100 without high Tris concentrations, and centrifuged again. The washed pellet containing the PhoV was suspended in buffer B, and the suspension was stirred overnight at room temperature. After centrifugation, the major part of the PhoV was present in the supernatant. This supernatant was subjected to ammonium sulphate fractionation. The $\mathrm{PhoV}$ precipitated in the $50-95 \%$ ammonium sulphate fraction, but was floating as a layer on top of the solution. This floating layer was redissolved in buffer $B$.

\section{RESULTS AND DISCUSSION}

\section{Cloning of an alkaline phosphatase-encoding gene from Synechococcus PCC 7942}

A gene coding for an APase was isolated from a Synechococcus PCC 7942 gene bank by screening for expression of APase activity in E. coli JM103, which expressed only a weak background of APase activity under the conditions used. About 17000 clones were grown on ampicillin-containing PA plates until tiny colonies were visible; the colonies were subsequently transferred onto nitrocellulose membranes. Cells were lysed, and non-bound cell debris was removed by several washing steps. The membranes were incubated in colonyassay buffer containing BCIP and NBT. By this chromogenic assay two clones were identified which strongly expressed APase activity. Plasmid DNA of both clones was isolated and characterized by restriction analysis. The hybrid plasmids contained inserts of $5.8 \mathrm{~kb}$ (pKW1) and $11.2 \mathrm{~kb}$ (pKW2), respectively. The $5.8 \mathrm{~kb}$ DNA fragment of plasmid pKW1 (Fig. 1) was part of pKW2 (data not shown) indicating that the two original clones encoded the same APase. Using both plasmids as probes in hybridization experiments with total DNA from Synechococcus PCC 7942 confirmed that no rearrangements or deletions had occurred during the cloning procedure (data not shown).

For further physical mapping of the APase-encoding gene within the $5.8 \mathrm{~kb}$ DNA fragment, hybrid plasmid pKW1 was partially digested with Sau3AI and subfragments were ligated into the Bam HI site of pUC19. Two hybrid plasmids ( $\mathrm{pKW} 4$ and $\mathrm{pKW}$ ) carrying subfragments that conferred APase activity are shown in Fig. 1. Plasmid pKW6 indicates that the maximum size of the APaseencoding gene was less than $2.5 \mathrm{~kb}$. Plasmids pKW4 and pKW6 contained the APase-encoding gene in different orientations relative to the lac promoter; thus it seems likely that the APase-encoding gene was transcribed from a promoter located within the cloned Synechococcus PCC
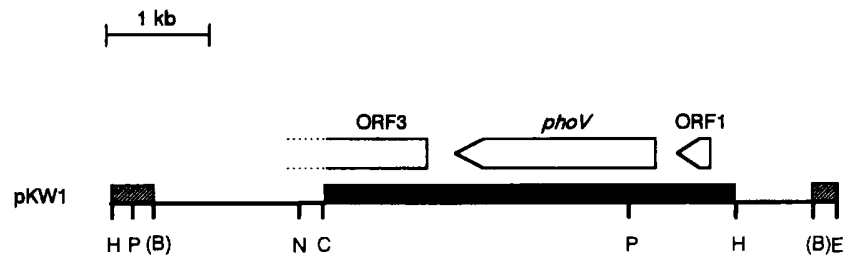

pKW4

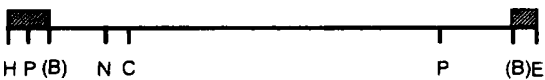

pKW6

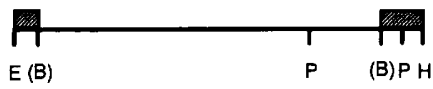

PKW13

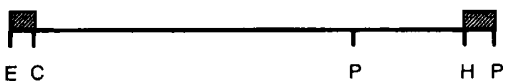

PKW14

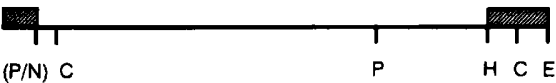

PKW14-A

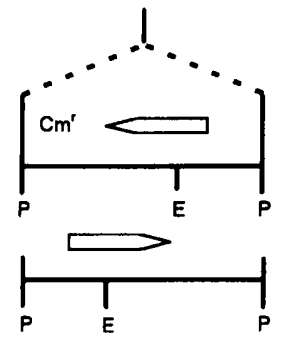

pKW14-B

Fig. 1. Physical and genetic map of the phoV gene region from Synechococcus PCC 7942. Plasmids pKW1, pKW4 and pKW6 contain different Sau3Al fragments (a $5.8 \mathrm{~kb}, 3.5 \mathrm{~kb}$ or $2.5 \mathrm{~kb}$ fragment, respectively) from Synechococcus PCC 7942 carrying the phoV gene cloned into the BamHI site of pUC19. Plasmids pKW13 and pKW14 contain subfragments of pKW1 cloned into the sequencing vector pSVB28. pKW13 contains a $3.3 \mathrm{~kb}$ ClalHindlll fragment from pKW1, and pKW14 contains a $3.5 \mathrm{~kb}$ NsilHindlll fragment from pKW1. Plasmids pKW14-A and pKW14-B contain the chloramphenicol resistance cassette $(\mathrm{Cm})$ used for mutational analysis. These plasmids are derivatives of pKW14 containing the $1.8 \mathrm{~kb}$ Pstl fragment from PRB5. The physical map of all hybrid plasmids is given for the enzymes BamHI (B), Clal (C), HindIII (H), Nsil (N) and Pstl (P). Restriction sites given in parentheses were destroyed during the cloning of DNA fragments with compatible ends which did not reconstitute the original sites. The hatched boxes represent the multiple cloning sites of pUC19 or pSVB28, and are not drawn to scale. The heavy line in pKW1 marks the DNA fragment which was sequenced.

7942 DNA fragment. However, this promoter recognized by $E$. coli is not necessarily the same promoter that drives expression in Synechococcus PCC 7942.

\section{DNA sequence analysis of the phoV gene region from Synechococcus PCC 7942}

The $3.3 \mathrm{~kb} \mathrm{ClaI}-\mathrm{HindIII}$ fragment and the $3.5 \mathrm{~kb} \mathrm{Nsi}$ HindIII fragment from plasmid $\mathrm{pKW} 1$ were cloned into the sequencing vector $\mathrm{pSVB} 28$. The resulting hybrid plasmids, pKW13 and pKW14, respectively (Fig. 1), were subjected to exonuclease III/S1 treatment to generate 
1 CATTGCAACTCAGAAAGCTAGGTTATAATTTAACTGACAAAGCAGTACTTGATGCCTTAAAAAGCGTAAAAAAAACTCAC

81 TGGCTTACAAGGTAGATGGCAAACGCTTCATAAAAAACCACCTTACCATTTGTGATACCGGACACAATAAAGCTGGCATC

$$
\begin{array}{llllllllllllllll}
M & V & I & G & M & V & K & D & K & D
\end{array}
$$

161 GCCGAAGTGGCACAAAACATCAAACAAACACCTTAATGAAAACCTACACATGGTGATTGGAATGGTCAAAGATAAAGATA

$\begin{array}{lllllllllllllllllllllllllllll}I & S & A & V & \text { L } & \text { A } & \text { L } & \text { L } & \text { P } & \text { A } & \text { N } & \text { A } & \text { N } & \text { Y } & \text { Y } & \text { F } & \text { C } & \text { Q } & \text { P } & \text { N } & \text { L } & \text { E } & R & \text { A } & \text { L } & \text { P } & \text { V }\end{array}$ 241 TTAGTGCTGTACTGGCCTTGTTGCCAGCCAACGCAAATTATTACTTTTGCCAGCCAAATTTAGAAAGGGCCCTACCTGTA

$\begin{array}{llllllllllllllllllllllllllllll}E & E & L & K & Q & Q & A & S & Q & Y & Q & L & R & G & E & A & F & T & S & G & S & D & A & L & Q & T & A\end{array}$ 321 GAGGAACTGAAACAGCAAGCCAGTCAATATCAACTCCGAGGCGAAGCGTTTACATCGGGTAGTGACGCTCTGCAAACTGC

\begin{tabular}{lllllllllllllllllllllll}
\hline & $S$ & $A$ & $A$ & $N$ & $E$ & $K$ & $D$ & $L$ & $I$ & $F$ & $I$ & $G$ & $G$ & $S$ & $T$ & $F$ & $V$ & $V$ & $A$ & $E$ & $I$ & $I$
\end{tabular}

401 GCAAAGTGCTGCTAACGAAAAGGATCTCATATTCATTGGTGGTAGCACATTTGTAGTAGCAGAAATTATATAAAATCTTT 481 ACAATGCGCACCAATTATCTTAACATCGTTTTAATACCTTTACAAAAACATCAATTTTATTAATGAAAATTAAACTTTTG $\begin{array}{llllllllllllllllllllllllllll}C & I & S & L & A & V & L & F & C & S & S & A \downarrow & N & A \downarrow & Q & K & K & Q & A & K & V & Q & P & S & V & F & P\end{array}$ 561 TGCATTTCTTTAGCTGTTTTGTTTTGCAGCAGTGCAAACGCCCAGAAAAAACAAGCTAAAGTTCAACCTTCTGTATTTCC

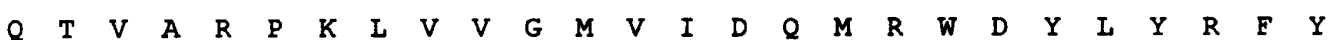
641 ACAAACGGTAGCTCGTCCCAAATTAGTAGTTGGGATGGTGATAGATCAAATGCGTTGGGATTATTTATATCGTTTTTATG

$\begin{array}{llllllllllllllllllllllllllll}A & R & Y & G & N & G & G & F & K & R & I & I & N & E & G & F & S & A & E & N & T & I & I & P & Y & T & P\end{array}$ 721 CCAGATATGGCAATGGTGGCTTCAAACGATTAATCAATGAAGGTTTTTCTGCAGAAAATACTTTGATCCCTTATACACCA PstI

$\begin{array}{lllllllllllllllllllllllllllllll}T & \text { I } & T & \text { A } & C & G & H & S & S & I & Y & T & G & S & V & P & A & I & N & G & I & I & G & N & N & * & W & F\end{array}$ 801 ACCTTAACTGCATGCGGCCACTCTTCAATTTATACTGGTTCTGTACCGGCTATTAATGGTATTATTGGCAATAACTGGTT

$\begin{array}{lllllllllllllllllllllllllll}D & P & Q & L & G & R & D & V & Y & C & V & E & D & K & S & V & K & T & V & G & S & S & S & N & E & G\end{array}$ 881 TGACCCTCAGTTGGGCAGGGATGTTTATTGTGTAGAAGATAAAAGCGTTAAAACCGTTGGAAGCTCAAGCAACGAAGGGC

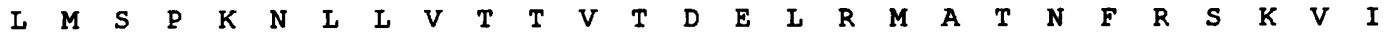
961 TAATGTCACCAAAAAATTTGCTAGTTACTACGGTAACCGACGAGCTTAGGATGGCCACCAACTTTAGAAGCAAGGTAATT

$\begin{array}{llllllllllllllllllllllllllll}S & V & S & I & K & D & R & G & A & I & L & P & G & G & H & T & A & N & G & A & Y & W & Y & D & D & M & T\end{array}$ 1041 AGTGTTTCTATTAAAGATAGAGGTGCGATTCTTCCTGGCGGGCATACGGCAAATGGCGCTTACTGGTACGATGACATGAC

$\begin{array}{llllllllllllllllllllllllllll}G & S & F & I & S & S & T & H & Y & M & Q & Q & L & P & T & W & V & N & D & F & N & A & Q & R & L & P\end{array}$ 1121 TGGCAGTTTTATTTCCAGCACGCATTACATGCAGCAATTGCCTACTTGGGTAAATGATTTTAATGCGCAAAGACTTCCCA

$\begin{array}{lllllllllllllllllllllllllllll}\mathbf{N} & \mathbf{K} & \mathbf{Y} & \mathbf{F} & \mathbf{E} & \mathbf{Q} & \mathbf{D} & \mathbf{W} & \mathbf{N} & \mathbf{T} & \mathbf{L} & \mathbf{Y} & \mathbf{P} & \mathbf{I} & \mathbf{E} & \mathbf{T} & \mathbf{Y} & \mathbf{T} & \mathbf{E} & \mathbf{S} & \mathbf{T} & \mathrm{A} & \mathrm{D} & \mathrm{A} & \mathrm{K} & \mathbf{P} & \mathbf{Y}\end{array}$ 1201 ATAAATACTTTGAACAAGATTGGATACCTTATATCCTATTGAAACTTATACGGAAAGTACCGCCGATGCAAAACCATAC

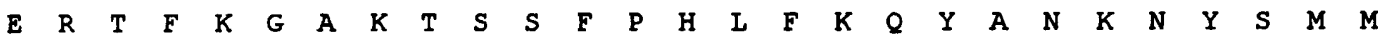
1281 GAGCGCACTTTTAAAGGTGCAAAAACCTCAAGTTTTCCTCACTTATTTAAACAATATGCTAATAAAAACTATTCGATGAT

$\begin{array}{llllllllllllllllllllllllll}A & S & M & P & Q & G & N & S & F & T & L & E & F & A & K & A & A & I & P & A & E & K & L & G & Q & T\end{array}$

1361 GGCAAGCATGCCACAGGGTAACAGCTTTACTTTAGAATTTGCCAAAGCTGCGATACCTGCTGAAAAACTTGGCCAAACTG

$\begin{array}{llllllllllllllllllllllllllll}G & N & T & D & F & \text { L } & A & V & S & L & S & S & T & D & Y & V & G & H & Q & F & G & P & N & S & I & E & L\end{array}$ 1441 GAAATACAGATTTCTTAGCAGTAAGTTTATCTTCAACAGATTATGTAGGGCACCAATTCGGACCAAATTCTATCGAGCTG

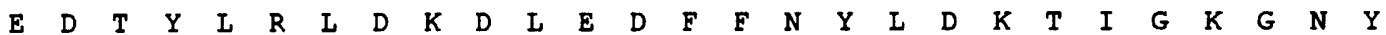
1521 GAAGATACGTATTTAAGGCTTGACAAAGATTTGGAAGATTTCTTCAACTATTTGGACAAAACCATCGGCAAAGGAAACTA

$\begin{array}{lllllllllllllllllllllllllll}L & I & F & \text { L } & T & \text { A } & D & H & G & A & T & H & V & P & G & F & L & R & N & K & M & P & G & G & R & L\end{array}$ 1601 TTTGTTATTCTTAACTGCCGACCACGGTGCAACTCATGTTCCTGGTTTTTTAAGAAACAAAATGCCTGGAGGTCGATTGC

$\begin{array}{lllllllllllllllllllllllllllll}\text { L } & I & K & V & Q & T & D & L & D & S & I & I & F & N & E & F & K & V & R & C & N & F & T & I & I & N & N\end{array}$ 1681 TACTAAAAGTTCAAACTGATTTAGATAGTTTGATTTTTAATGAGTTTAAAGTGAGATGTAATTTCACCATTATTAACAAT

$\begin{array}{lllllllllllllllllllllllllll}Q & V & I & F & D & T & D & A & I & K & E & A & K & A & D & Y & A & K & I & K & Q & S & T & I & D & Y & I\end{array}$ 1761 CAAGTGATTTTTGATACTGATGCAATTAAGGAAGCTAAAGCTGATTATGCTAAAATCAAACAATCCACGATAGATTATTT

$\begin{array}{llllllllllllllllllllllllllllllll}V & K & Q & D & G & V & I & N & A & V & D & I & K & N & M & G & A & V & T & I & P & Q & E & I & K & N\end{array}$ 1841 GGTAAAACAAGACGGGGTTTTAAATGCGTAGACATTAAAAATATGGGTGCAGTTACTATCCCTCAAGAAATTAAAAATA $\begin{array}{llllllllllllllllllllllllllll}K & I & I & N & G & Y & N & A & R & R & S & G & D & V & Y & I & I & \text { L } & \text { D } & A & G & \text { W } & \text { Y } & \text { P } & \text { T } & \text { L } & T\end{array}$ 1921 AAATCATCAATGGGTATAATGCCCGTAGAAGTGGCGACGTATATATCATTCTCGATGCTGGCTGGTATCCTACTTTAACT

Fig. 2. For legend see facing page. 
$\begin{array}{llllllllllllllllllllllllllllllll}\text { P } & G & \text { T } & G & H & A & A & W & N & P & Y & D & S & H & I & P & A & L & F & M & G & W & G & V & K & P & G\end{array}$ 2001 CCAGGCACAGGGCATGCCGCATGGAATCCTTACGATAGTCACATTCCTGCACTTTTCATGGGTTGGGGCGTTAAACCTGG

$\begin{array}{llllllllllllllllllllllllllllll}K & T & N & K & E & Y & Y & M & S & D & I & A & P & T & V & S & A & L & I & H & I & Q & Q & P & S & G\end{array}$

2081 TAAAACAAATAAAGAATATTATATGTCCGATATTGCCCCTACTGTTTCTGCCTTGTTGCATATTCAACAACCAAGCGGTA

$\begin{array}{llllllllllll}S & I & G & K & V & I & T & D & L & L & K\end{array}$

2161 GTATAGGCAAAGTAATAACCGATTTACTTAAATAGCTTTAAAATTTTAATAATTCAACCCTCATTTCTGAAATATGAATT

2241 GAGGGTTTTTCATTATTAGGGCTATTTTAATACCAGATCTTCAAATTTACTACACATTTTTACTCCTAAAATTTAGCGTT

2321 TTCACCTAGCAAAAATCTTACTTTGGTACATATCTATTAGCCAAATATTAGCAAAAATTATATCAGGATTTATCGTATGG

ORF3

$\begin{array}{llllllllllllllllllllllllllll}D & K & I & E & I & Y & Q & P & K & H & K & I & R & F & V & T & V & A & A & L & F & D & G & H & D & A & T\end{array}$

2401 ATAAAATTGAAATCTATCAGCCTAAACATAAAATAAGATTTGTAACTGTCGCTGCACTTTTTGATGGGCATGATGCTACC

$\begin{array}{llllllllllllllllllllllllllll}I & N & I & M & R & R & I & I & Q & S & S & G & A & E & V & I & H & I & G & H & N & R & S & V & E & E & V\end{array}$ 2481 ATTAATATCATGCGTAGAATTTTGCAGTCCAGCGGTGCCGAGGTTATTCACTTAGGGCATAACCGTTCGGTGGAAGAAGT

$\begin{array}{lllllllllllllllllllllllllllllllllll}V & N & C & A & I & Q & E & D & V & Q & G & I & A & M & T & S & Y & Q & G & G & H & I & E & Y & F & K\end{array}$

2561 TGTAAATTGCGCAATACAAGAAGATGTTCAAGGTATTGCGATGACATCTTATCAGGGTGGACACATTGAATACTTTAAAT

$\begin{array}{llllllllllllllllllllllllllll}Y & M & Y & D & \text { L } & \text { L } & Q & Q & E & R & G & A & N & H & I & K & I & F & G & G & G & G & G & V & I & L & P & N\end{array}$

2641 ATATGTATGATTTATTGCAGGAACGTGGTGCAAATCATATCAAAATATTTGGTGGTGGAGGTGGTGTAATTTTACCTAAT

$\begin{array}{llllllllllllllllllllllllllllll}E & I & K & E & L & E & S & Y & G & I & A & E & I & I & F & A & D & D & G & R & R & I & G & T & Q & G & M\end{array}$

2721 GAAATTAAAGAACTAGAGAGCTACGGTATTGCGGAGATCTTATTCGCGGACGACGGTAGAAGAATTGGGACGCAAGGCAT

$\begin{array}{lllllllllllllllllllllllllllll}I & N & D & M & L & Q & K & N & R & F & C & I & I & E & K & Q & F & D & L & E & K & P & I & N & R & E\end{array}$

2801 GATTAACGACATGCTTCAAAAAAACAGATTTTGCATTATAGAAAAGCAATTCGATTTAGAAAAGCCATTAAATAGGGAGA

$\begin{array}{llllllllllllllllllllllllllll}T & T & E & D & R & K & R & N & L & F & G & R & N & N & A & E & A & F & E & D & S & I & A & T & I & R & K\end{array}$

2881 CAACGGAAGATCGCAAACGCAATCTCTTTGGCAGAAATAATGCCGAAGCCTTTGAAGACTCGTTAGCAACGCTGAGAAAA

$\begin{array}{lllllllllllllllllllllllllllllll}K & Q & P & T & N & P & P & V & L & G & I & T & G & T & G & G & A & G & K & S & S & L & V & D & E & L & V\end{array}$ 2961 AAACAGCCTACCAACCCACCTGTTTTAGGTATTACCGGAACAGGCGGCGCAGGAAAGTCTTCCTTAGTTGACGAATTGGT

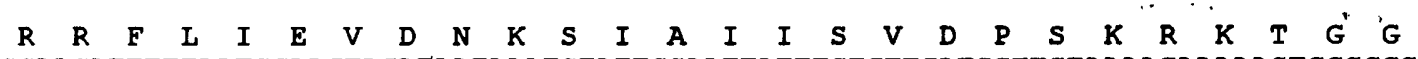

3041 TCGAAGATTTTTAATCGAAGTAGATAATAAATCTATTGCAATTATTTCTGTTGATCCTTCTAAAAGAAAAACTGGCGGCG

$\begin{array}{llllllllllllllllllllllllllll}A & L & L & G & D & R & I & R & M & N & A & I & N & N & P & R & V & Y & M & R & S & L & A & T & R & Q & A\end{array}$

3121 CCTTGTTGGGAGATAGAATAAGAATGAATGCCATTAACAATCCAAGGGTTTATATGCGTTCATTAGCTACTAGGCAAGCT

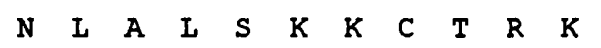

3201 AACCTCGCCCTATCTAAAAAATGTACAAGAAAGC

Fig. 2. Nucleotide sequence of the Synechococcus PCC 7942 phoV gene region. The DNA sequence is presented in the $5^{\prime} \rightarrow 3^{\prime}$ direction. The predicted amino acid sequences for two complete open reading frames (ORF1 and PhoV) and for one incomplete open reading frame (ORF3) are indicated by the single-letter code. Potential cleavage sites for a secretory leader sequence are indicated by vertical arrows. The Pstl site within the phoV coding region used for cassette mutagenesis is shown below the nucleotide sequence. A putative phosphorylation site of PhoV is underlined.

nested deletions. The deletion derivatives were used to determine the sequence of a contiguous 3234 bp DNA fragment in overlapping sequences for both strands (Fig. 2).

Three ORFs within the sequenced region could be defined in the same orientation. ORF1 might encode a small polypeptide of 87 amino acid residues, but computer searches did not reveal any homology to known genes. Since hybrid plasmid pKW6 (Fig. 1) did not contain the entire ORF1 but still confers APase activity, at least in E. coli ORF1 is not essential for APase activity.

ORF2 was located 72 bp downstream of ORF1 and codes for a protein of 550 amino acid residues with a calculated molecular mass of $61325 \mathrm{Da}$ and an isoelectric point of
8.7. There are several lines of evidence that ORF2 corresponds to the APase-encoding gene from Synechococcus PCC 7942. (i) ORF2 was the only complete ORF encoded by the insert in pKW6, which confers APase activity. (ii) Insertion of an interposon carrying the chloramphenicol resistance gene in the PstI site within ORF2 (Fig. 1) abolished APase activity in E. coli strains carrying the plasmid (see below). (iii) ORF2 showed $34 \%$ identity over its entire length with the $p h o A$ gene product from Zymomonas mobilis, strain CP4 (database accession number L36230; P. F. Gomez \& L. O. Ingram, unpublished, 1994) (Fig. 3). Since another phosphataseencoding gene from Synechococcus PCC 7942 has been described as pho $A$ (Ray et al., 1991), the ORF2 gene was called $p h o V$. 
(a)

SphoV

ZphoA

SphoV

ZphoA

sphov

ZphoA

Sphov

ZphoA

Sphov

ZphoA

sphov

ZphoA

sphov

ZphoA

Sphov

ZphoA

Sphov

IphoA

Sphov

ZphoA

(b)

S-ORF 3

M55343

S-ORF3

M55343

S-ORE3

M55343

S-ORF

M55343 ITVLE

S-ORF3 IISVDPSKRKTGGALLGDRIRMNAINNPRVYMRSIATRQANLALSKKCTRK

MDKIEIYQPKHKIREVTVAALFDGHDATINIMRRILQSSGAEVIHLGH

NRSVEEVVINCAIQEDVQGIAMTSYQGGHIEYFKYMYDLLQERGANHI KIFGGGGGVILPN

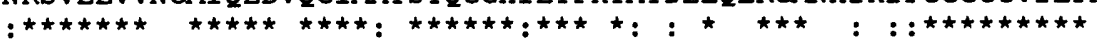
DRSVEEVVTAAIQEDAQGIALSSYQGGHVEYFSYLIERLRARGAGNVRVYGGGGGVILPA

EIKELESYGIAEILFADDGRRIGTQGMINDMLQKNRFCI IEKQFDLEKPLNRETTEDRKR

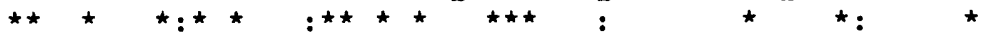
EIDLLHARGVARI FSPQDGQRLGLAKMINMI I EACDVDLTEDGPKLDAVAAGEHGALARA
MKIKLLCISIAVLFCSSANAOKKOAKVOPSVFPOTVARP . . . K

$$
: * \quad * \star \quad \text { * : } * \text { * }
$$

40

MNSLLHHSFLKTVESSLAIAIVTSSLSSVTIAATHPLDNHPKGEIAASSETAHNPWSGTR

LVVGMVIDQMRWDYLYRFYARYGNGGFKRLINEGFSAENTLI PYTPTLTACGHSS IYTGS

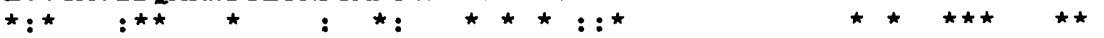

LIVAISVDQFSSDLFSEYRGRF . RSGMKQL . QNGVVYPMAYHSHAATETCPGHSVLLTGD

VPAINGI IGNNWFD. . . PQLGRDVYCVEDKSVKTVGSSSNEGLMSPKNLLVTTVTDELRM

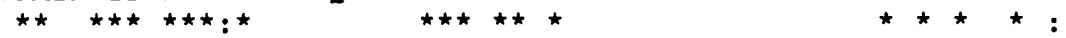
HPARTGI IANNWYDESVKRADKKVYCSEDPSLSADPQNYKPSV . . . HYLKVPTLGDRMKK

ATNFRSKVISVSIKDRGAILPGGHTANGA. YWYDDMTGSFISSTHYMQQLPTWVNDFNAQ

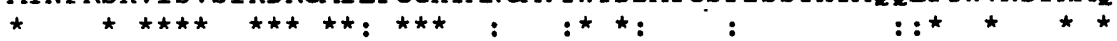
ANP . HSRVISVAGKDRAAIMMGGHMTDQIWFWSDN . . . AYKTLADHKGEMPATVKTVNEQ RLPNKYFEQDWNTLYPIETYTESTADAKPYERTFKGAKTSSFPHLFKQYANKNYSMMASM $: \star \star * \star * * * *$ * $*$ : . . VTRLMQQDEAPVMPSVCADHASALKI GNNRI I GLAPASRKTGDFKT ERVT . . . . . . . PQGNS FTLEFAKAAI PAEKLGQTGNTD FLAVSLSSTDYVGHQFGPNS IELEDTYLRLDKD

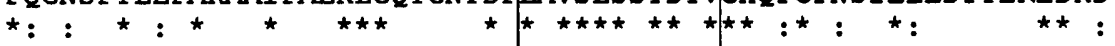
PDYDRTTTDIAIGLIDELKLGHGNAPDLLTVSLSATDAVGHAYGTEGAEMCSQMAGLDDN LEDFFNYLDKTIGKGNYLLFLTADHGATHVPGFLRNKMPGGRLLLKVQTDLDSLIFNEFK

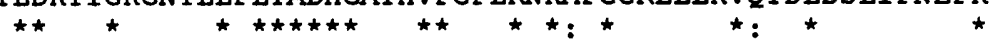
IARI IAALDSN. GVPY. VLVLTADHGGQDVPE. . RAKLRGVETAQRVDPALSPDQLS . LK VRCNFTI INNQVIF . . . . . . . . DTDAIKEAKADYAKIKQSTIDYLVKQDGVLNAVDI LAERFQLSHNQPLFFANEPQGDWYINRNLPEQTKAOLIQAAKSELSNHPQVAAVFTASEL KNMGAVT I PQEI . . . KNKI INGYNARRSGDVYI ILDAGWYPTLTP . . . GTGHAAWNPYD

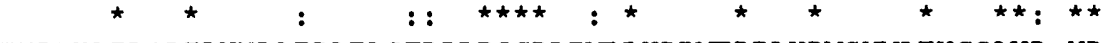
THI PHPTRSPELWNLAERAEASFDPLRSGDLIVLLKPRVT PIAKPVSYVATHGSAWD. YD SHI PALFMGWGVKPGKTNKEYYMSDIAPTVSALLHIQQPSGSIGKVITDL . . . . . . . LK

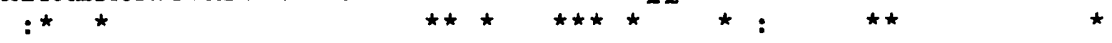
RRVPI IFYTPHASGFEQPMPVETVDIMPSLAALIQI PLRKGEVDGRCLDLDPTEATTCPVK

100 118 157 175 216 231 276 281 336 341 396 396 445 456 498 515 550 576

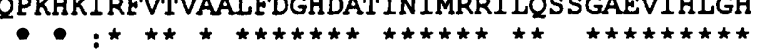

Fig. 3. Alignment of predicted amino acid sequences of PhoV and ORF3 from Synechococcus PCC 7942 (S-PhoV and SORF3) and analogous proteins from Zymomonas mobilis (Z-PhoA) and Frankia sp. (F-ORF). The amino acid sequences are aligned for maximum matching and identical amino acid residues are marked by asterisks. Conservative substitutions are marked by colons. The putative phosphorylation site in S-PhoV and Z-PhoA is boxed. (a) Alignment of S-PhoV from Synechococcus PCC 7942 (this paper; database accession number Z48801) and Z-PhoA from Z. mobilis strain CP4 (database accession number L36230; P. F. Gomez \& L. O. Ingram, unpublished 1994). (b) Alignment of S-ORF3 from Synechococcus PCC 7942 (this paper; database accession number 248801) and F-ORF from Frankia sp. (database accession number M55343; Normand et al., 1992). 
Analysis of the deduced amino acid sequence of the Synechococcus PCC 7942 pho $V$ gene product using the PC/GENE programs revealed the presence of a prokaryotic leader peptide involved in secretion. Two potential cleavage sites were identified, either between positions 18 and 19 or between positions 20 and 21 (see Fig. 2). These predictions are in good agreement with the localization of the pho $V$ gene product in the periplasmic space of $E$. coli strains carrying plasmid $\mathrm{pKW} 1$ (see below). In addition, a putative phosphorylation site that is conserved between PhoV of Synechococcus PCC 7942 and PhoA of Z. mobilis was identified in the central part of the APase (marked in Fig. 3). However, an involvement of this site in regulation of APase activity remains speculative.

The third open reading frame (ORF3: Figs 1 and 2) was separated by $204 \mathrm{bp}$ from the $p h o V$ gene. ORF3 was incomplete in the subclones of pKW1 (Fig. 2), since the coding region exceeded the sequenced region of $\mathrm{pKW} 13$ at its $3^{\prime}$ end. A high degree of identity $(57 \%)$ was found for the N-terminal part of the deduced ORF3 gene product and a gene product from Frankia sp. (database accession number M55343; Normand et al., 1992). The corresponding gene from Frankia sp. is associated with rRNA genes, but the exact function of the gene product is not known.

\section{Mutational analysis of the Synechococcus PCC 7942 phoV gene}

To analyse the function of the phoV gene product in Synechococcus PCC 7942 we attempted to construct a null mutant. For this purpose a $1.8 \mathrm{~kb}$ Pst I fragment carrying a chloramphenicol resistance gene $\left(\mathrm{Cm}^{\mathrm{r}}\right)$ was isolated from hybrid plasmid pRB5 (Bockholt et al., 1991) and ligated into the single Pst $\mathrm{I}$ site within the phoV coding region. The $\mathrm{Cm}^{\mathrm{r}}$ cassette was inserted in both orientations into plasmid $\mathrm{pKW} 14$; the resulting hybrid plasmids pKW14-A and pKW14-B are shown in Fig. 1. In contrast to plasmid pKW14, hybrid plasmids pKW14-A and pKW14-B no longer expressed APase activity in E. coli JM103.

The $\mathrm{Cm}^{\mathrm{r}}$ cassette had previously been demonstrated to be useful in the construction of a Synecbococcus PCC $7942 p s b O$ null mutant (Bockholt et al., 1991). Therefore, plasmid pRB5 carrying the $p s b O::\left[\mathrm{Cm}^{\mathrm{r}}\right]$ mutant gene was used as a control during the attempts to construct a $p h o V$ null mutant. Transformation of Synechococcus PCC 7942 wildtype with pRB5 yielded about $52 \mathrm{Cm}^{\mathrm{r}}$ mutants per $\mu \mathrm{g}$ plasmid DNA. Since Synechococcus PCC 7942 has a very effective recombination system, more than $80 \%$ of the $\mathrm{Cm}^{\mathrm{r}}$ mutants had lost the vector-encoded ampicillin resistance due to double recombination events (Bockholt et al., 1991). However, transformation of Synechococcus PCC 7942 with either pKW14-A or pKW14-B did not lead to $\mathrm{Cm}^{\mathrm{r}}$ mutants. Even merodiploid mutants carrying pKW14-A or $\mathrm{pKW} 14-\mathrm{B}$ integrated into the chromosome due to single recombination events were not viable. The most likely explanation for this result is that the $3.5 \mathrm{~kb}$ HindIII-NsiI fragment from pKW14 belongs to one transcriptional unit and one gene of this putative operon (either $p h o V$ or ORF3) is essential in Synechococcus PCC 7942. A single crossover would have regenerated a wildtype $p h o V$ copy and a truncated ORF3 plus the mutated phoV gene and an eventually untranscribed wild-type ORF3 which would indicate that ORF3 is required. However, this assumption does not explain why we did not find $\mathrm{Cm}^{\mathrm{r}} \mathrm{Ap}^{\mathrm{r}}$ transformants which were not completely segregated and retained at least one wild-type copy (of the approximately 10 copies) of the genome to maintain viability. Since the pho $A$ gene of $Z$. mobilis (ZM1) also could not be inactivated (Michel et al., 1992), it seems likely that both Synecbococcus PCC 7942 PhoV and Z. mobilis ZM1 PhoA might be essential for viability. However, from the information available in the literature, it is not clear whether the APase from Z. mobilis CP4 (database accession number L36230; P. F. Gomez \& L. O. Ingram, unpublished, 1994), which shows $34 \%$ identity to the Synechococcus PCC $7942 \mathrm{PhoV}$, is identical with the APase isolated and characterized from $Z$. mobilis ZM1 (Michel \& Baratti, 1989; Michel et al., 1992; Baoudene-Assali et al., 1993).

\section{Expression of the Synechococcus PCC 7942 PhoV in E. coli CC118(pKW1)}

For partial purification and characterization of the Synechococcus PCC 7942 PhoV, the APase-free E. coli strain CC118 (Manoil \& Beckwith, 1985) was transformed with pKW1. In extracts of the transformed cells the detectable phosphatase activity corresponded to $1.7 \mu \mathrm{mol} 4-\mathrm{NP}$ formed (mg protein) ${ }^{-1} \mathrm{~min}^{-1}$ (as compared to zero activity when E. coli CC118 was transformed with pUC19). When the $E$. coli cells were transferred to phosphate-free medium, no further increase in phosphatase actvity was obtained during a growth period of $24 \mathrm{~h}$. This result implies that in E. coli CC118(pKW1) the expression of the Synechococcus PCC 7942 PhoV was not phosphateregulated. For comparison, the expression of the phosphate-regulated $E$. coli PhoA in wild-type cells ( $E$. coli JM103) was examined. Within $24 \mathrm{~h}$ under our incubation conditions in phosphate-free medium, the activity of the E. coli PhoA increased approximately 100 fold [from 0.02 to $2.50 \mu \mathrm{mol} 4-\mathrm{NP}$ formed (mg protein) ${ }^{-1}$ $\min ^{-1}$. It should be pointed out that the activity of the Synechococcus PCC 7942 PhoV in E. coli CC118(pKW1) was almost as high as the activity of the E. coli PhoA in E. coli JM103 under phosphate deficiency: 1.7 vs $2.5 \mu \mathrm{mol} 4-\mathrm{NP}$ formed (mg protein) ${ }^{-1} \mathrm{~min}^{-1}$, respectively.

\section{Localization of the Synechococcus PCC 7942 PhoV in E. coli CC118(pKW1)}

After applying the osmotic shock procedure commonly used to disrupt the outer cell membrane of E. coli (Neu \& Heppel, 1965), APase activity was found in the spheroplast fraction. No, or only minor, activity was detectable in the soluble periplasmic protein fraction. Since 4-NPP cannot penetrate the cytoplasmic membrane of spheroplasts, it can be concluded that the recombinant Synechococcus PCC 7942 PhoV was extracellular and membrane-bound. It was most likely associated with the 
Table 1. Partial purification of the Synechococcus PCC 7942 PhoV from E. coli CC118(pKW1)

Details of the purification procedure are given in Methods.

\begin{tabular}{|c|c|c|c|}
\hline \multirow[t]{2}{*}{ Purification step } & \multirow{2}{*}{$\begin{array}{c}\text { Total } \\
\text { protein } \\
\text { (mg) }\end{array}$} & \multicolumn{2}{|c|}{ Phosphatase activity* } \\
\hline & & $\begin{array}{c}\text { Total } \\
\text { (units) }\end{array}$ & $\begin{array}{l}\text { Specific activity } \\
\text { [units (mg } \\
\text { protein) })^{-1} \text { ] }\end{array}$ \\
\hline \multicolumn{4}{|l|}{ French press extract: } \\
\hline Total & 4808 & 8525 & $1 \cdot 8$ \\
\hline Pellet & 2640 & 6339 & $2 \cdot 4$ \\
\hline Supernatant & 2117 & 2175 & $1 \cdot 0$ \\
\hline \multicolumn{4}{|l|}{ Extraction of pellet with buffer $\mathrm{B}+$ : } \\
\hline APase activity in supernatant & 432 & 2010 & $4 \cdot 7$ \\
\hline \multicolumn{4}{|l|}{ Dialysis of extract against buffer $A \dagger$ : } \\
\hline APase activity in pellet & 340 & 1822 & $5 \cdot 4$ \\
\hline \multicolumn{4}{|l|}{ Re-extraction of washed pellet with buffer $\mathrm{B}+$ : } \\
\hline APase activity in supernatant & 112 & 1049 & $9 \cdot 4$ \\
\hline Ammonium sulphate fractionation $(50-95 \%)$ & 34 & 758 & $22 \cdot 2$ \\
\hline
\end{tabular}

$* 1$ unit of enzyme corresponds to $1 \mu \mathrm{mol} 4-\mathrm{NP}$ formed $\min ^{-1}$ (at $37^{\circ} \mathrm{C}$ ).

† Buffer A: $10 \mathrm{mM}$ Tris/HCl, $\mathrm{pH} 7 \cdot 6$, containing $1 \mathrm{mM} \mathrm{MgCl}$ and $1 \mathrm{mM} \mathrm{ZnCl}$. Buffer B: $1 \mathrm{M} \mathrm{Tris} / \mathrm{HCl}$, $\mathrm{pH} 7 \cdot 6$, containing $10 \mathrm{mM} \mathrm{MgCl}_{2}, 10 \mathrm{mM} \mathrm{ZnCl}_{2}$ and $2 \%$ Triton X-100.

periplasmic side of the inner membrane in E. coli CC118(pKW1) (results not shown). A periplasmic location of the PhoV is in agreement with the presence of a prokaryotic leader peptide involved in secretion (Fig. 2).

\section{Extraction and partial purification of the Synechococcus PCC 7942 PhoV from E. coli CC118(pKW1)}

The membrane-associated PhoV enzyme was solubilized from E. coli CC118(pKW1) with Triton X-100 in concentrated Tris buffer $(1 \mathrm{M}$ Tris $/ \mathrm{HCl}, \mathrm{pH} 7 \cdot 6$, containing $10 \mathrm{mM} \mathrm{MgCl}_{2}, 10 \mathrm{mM} \mathrm{ZnCl}_{2}$ and $\%$ Triton X-100). For optimal extraction a high Tris concentration, in addition to the presence of Triton X-100, was necessary. We were not successful in isolating PhoV in a homogeneous form. However, a partial purification was achieved by a differential extraction procedure as described in Methods. The results of such a partial purification are given in Table 1 and show that an approximately 10-fold increase in specific activity was obtained. This partially purified PhoV fraction contained rather low quantities of proteins in the $40-100 \mathrm{kDa}$ region except the PhoV (61 kDa), but was greatly enriched in low molecular mass proteins of 10-30 kDa (not shown). The fact that the PhoV was recovered as a floating layer after ammonium sulphate fractionation implies that it may be a lipoprotein. Column chromatography for further purification was unsuccessful because of aggregation, low solubility and inactivation problems when the Tris concentration was reduced to allow chromatography on ion-exchange columns. Chromatography on hydrophobic column materials was also unsuccessful. A successful purification will most likely require empirical testing of several different ionic detergents to find one which will keep the PhoV in solution in the absence of high Tris concentrations; such an approach was recently successful in purifying the PhoA from Z. mobilis (Baoudene-Assali et al., 1993).

\section{Preliminary characterization of the Synechococcus PCC 7942 PhoV}

A preliminary enzymic characterization was performed with the enriched PhoV fraction. APase activity on 4NPP was observed between $\mathrm{pH} 6$ and $\mathrm{pH} 11$, with optimal activity in the broad $\mathrm{pH}$ range of $7-10$. These results are in agreement with the $\mathrm{pH}$ range reported for other APases in bacteria. APase activity increased with incubation temperature between 15 and $35^{\circ} \mathrm{C}$ and decreased upon further heating; no activity was observed at $65^{\circ} \mathrm{C}$. Preincubation of the PhoV for $10 \mathrm{~min}$ at 50 or $60{ }^{\circ} \mathrm{C}$ caused $50 \%$ or $100 \%$ inactivation, respectively. Renaturation of the heat-inactivated $\mathrm{PhoV}$ was not possible. Thus, in contrast to several of the wellcharacterized APases which have been shown to be fairly heat resistant (Von Tigerstrom \& Stelmaschuk, 1986; Gaten \& Levinthal, 1960), PhoV is fairly heat-labile. Heat-labile APases have seldom been described in the literature. The APase of Antarctic bacteria was inactivated by incubation for $10 \mathrm{~min}$ at $55^{\circ} \mathrm{C}$ (Kobori et al., 1984). The PhoA of $Z$. mobilis $Z M 1$ remained stable for $1 \mathrm{~h}$ at $60^{\circ} \mathrm{C}$, but further increase in temperature caused inactivation (Michel \& Baratti, 1989).

Optimal activity of the Synechococcus PCC 7942 PhoV was dependent on the presence of Triton X-100 in the reaction mixture. Triton X-100 at a concentration of $0.05 \%$ increased the activity about fivefold relative to that in its 
Table 2. Substrate activity

APase activity on various phosphomonoesters was determined as described in Methods. The specific activity obtained with 4-NPP was set as $100 \%$.

\begin{tabular}{|lcc|}
\hline Phosphomonoester & $\begin{array}{c}\text { Relative } \\
\text { specific activity } \\
\mathbf{( \% )}\end{array}$ & $\begin{array}{c}\boldsymbol{K}_{\mathbf{m}} \\
\mathbf{( m M )}\end{array}$ \\
\hline 4-NPP & 100 & $0 \cdot 1$ \\
ATP & 41 & $1 \cdot 0$ \\
ADP & 33 & 0.5 \\
AMP & 29 & 1.5 \\
D-Glucose 6-phosphate & 48 & 1.9 \\
Fructose 1,6-phosphate & 30 & $1 \cdot 4$ \\
Fructose 6-phosphate & 19 & 2.3 \\
Fructose 1-phosphate & 62 & 3.8 \\
3-Phosphoglycerate & 50 & 1.9 \\
3-Phosphocreatin & 136 & $2 \cdot 4$ \\
\hline
\end{tabular}

absence. Maximum activity was obtained with Triton $\mathrm{X}$ 100 in a concentration range of $0.01-2 \%$. The enzyme was even active in Triton X-100 concentrations up to $80 \%$, indicating a particular resistance to Triton X-100.

PhoV has a broad substrate specificity (Table 2) and thus can be classified as a nonspecific phosphomonoesterase. The lowest $K_{\mathrm{m}}$ values were obtained for ADP, ATP and 4-NPP, while the highest relative specific activities were observed with phosphocreatine and 4-NPP. As for other APases, phosphate was a competitive inhibitor of the reaction, with a $K_{\mathrm{i}}$ value of $0.57 \mathrm{mM}$. The enzyme was sensitive to chelators, such as EDTA ( $50 \%$ inhibition at $4 \mathrm{mM}$ EDTA) or citrate $(50 \%$ inhibition at $48 \mathrm{mM}$ sodium citrate), a common feature of most APases (McComb et al., 1979).

After dialysing the PhoV preparation against EDTA, $\mathrm{ZnCl}_{2}$ addition was required to obtain maximal activity, implying that $\mathrm{Zn}^{2+}$ was partially lost by the EDTA treatment (Fig. 4). $\mathrm{ZnCl}_{2}$ increased $\mathrm{PhoV}$ activity up to a concentration of about $1 \mathrm{mM}$. Further increase in $\mathrm{ZnCl}_{2}$ concentration caused a drastic reduction of activity, an observation made for several phosphatases (Cathala \& Brunel, 1975). At suboptimal $\mathrm{Zn}^{2+}$ concentrations $\mathrm{MgCl}_{2}$ was stimulatory, but in contrast to $\mathrm{ZnCl}_{2}, \mathrm{MgCl}_{2}$ concentrations up to $0.2 \mathrm{M}$ were not inhibitory. $\mathrm{MgCl}_{2}$ caused no further increase in activity when the $\mathrm{ZnCl}_{2}$ concentration was optimal ( $1 \mathrm{mM} \mathrm{ZnCl}{ }_{2}$ ). Other divalent cations, such as $\mathrm{Ca}^{2+}$ or $\mathrm{Co}^{2+}$, had essentially no effect on the $\mathrm{PhoV}$ actvity. $\mathrm{Mn}^{2+}$ was strongly inhibitory, giving $50 \%$ and $100 \%$ inhibition at $0 \cdot 1$ and $1 \mathrm{mM} \mathrm{MnCl}$, respectively. Thus, like several other APases, PhoV requires $\mathrm{Zn}^{2+}$ for activity, and $\mathrm{Mg}^{2+}$ is stimulatory. Inhibition by high $\mathrm{Zn}^{2+}$ concentrations might be caused by $\mathrm{Zn}^{2+}$ binding to the $\mathrm{Mg}^{2+}$ site (see, for example, Cathala \& Brunel, 1975). For several APases, such as bovine kidney APase (Cathala \& Brunel, 1975), $\mathrm{Mn}^{2+}$ was shown to be stimulatory, replacing $\mathrm{Mg}^{2+}$. However, $\mathrm{Mn}^{2+}$ was strongly inhibitory

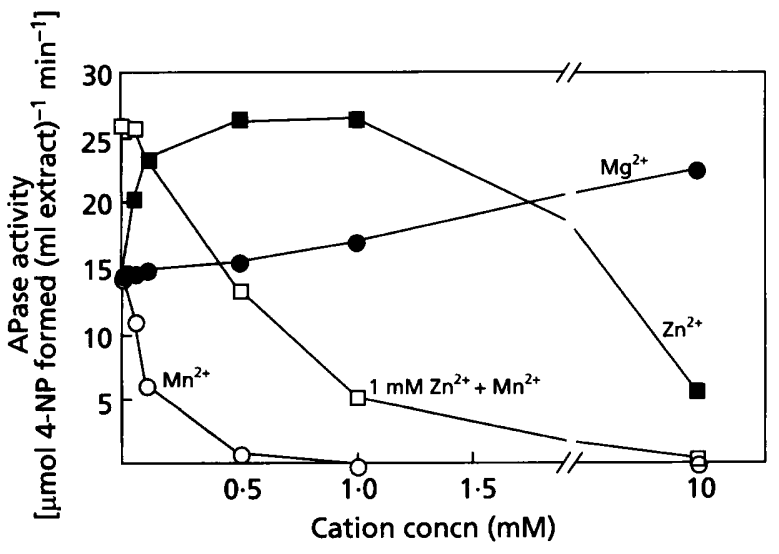

Fig. 4. Influence of various cations on the Synechococcus PCC 7942 PhoV activity. The partially purified PhoV preparation was dialysed against $10 \mathrm{mM}$ Tris/ $/ \mathrm{HCl}, \mathrm{pH} 7 \cdot 6$, containing $10 \mathrm{mM}$ EDTA. APase activity was determined as described in Methods with the modification that cations (added as $\mathrm{ZnCl}_{2}, \mathrm{MgCl}_{2}$ or $\mathrm{MnCl}_{2}$ ) were present in the reaction mixture as indicated in the figure.

to the Synechococcus PCC 7942 PhoV, most likely by replacing the essential $\mathrm{Zn}^{2+}$ from its specific binding site.

\section{Concluding remarks}

Based on the results here presented it can be concluded that Synechococcus PCC 7942 has at least two APases. One APase is phosphate-repressible (PhoA, $145 \mathrm{kDa}$ ) (Block \& Grossman, 1988; Ray et al., 1991), while the one here described is most likely not repressed by phosphate (PhoV, 61.3 kDa). The presence of a second APase in Synechococcus PCC 7942 could be important in providing the cells with sufficient phosphate under stress conditions, since Ray et al. (1991) speculated that the atypical $145 \mathrm{kDa}$ PhoA might have functions in addition to hydrolysing phosphomonoesters. Similar results with respect to the presence of two APases have been obtained with Bacillus subtilis, which contains a phosphate-repressible and a phosphate-nonrepressible APase (Hulett et al., 1990). Elucidation of the exact function of the PhoV in Synechococcus PCC 7942, and of the regulation of its expression and activity (especially since a regulation by phosphorylation/dephosphorylation seems possible) requires further work.

\section{ACKNOWLEDGEMENTS}

The financial support of Boehringer Mannheim $\mathrm{GmbH}$ is gratefully acknowledged. K.-U. Wagner wishes to thank the Studienstiftung des Deutschen Volkes for his fellowship.

\section{REFERENCES}

Arnold, W. \& Puhler, A. (1988). A family of high-copy-number plasmid vectors with single end-label sites for rapid nucleotide sequencing. Gene 70, 171-179.

Baoudene-Assali, F., Baratti, J. \& Michel, G. P. F. (1993). Purification and properties of a phosphate-irrepressible membrane- 
bound alkaline phosphatase from Zymomonas mobilis. J Gen Microbiol 139, 229-235.

Bhatti, A. R., De Voe, I. W. \& Ingram, J. M. (1976). The release and characterization of some periplasm-located enzymes of Pseudomonas aeruginosa. Can J Microbiol 22, 1425-1429.

Block, M. A. \& Grossman, A. R. (1988). Identification and purification of a derepressible alkaline phosphatase from Anacystis nidulans R2. Plant Pbysiol 86, 1179-1184.

Bockholt, R., Masepohl, B. \& Pistorius, E. K. (1991). Insertional inactivation of the $p s b \mathrm{O}$ gene encoding the manganese stabilizing protein of photosystem II in the cyanobacterium Synechococcus PCC7942: effect on photosynthetic water oxidation and L-amino acid oxidase activity. FEBS Lett 294, 59-63.

Bone, D. H. (1971). Relationship between phosphates and alkaline phosphatase of Anabaena flos-aquae in continuous culture. Arch Microbiol 80, 147-153.

Bradford, M. M. (1976). A rapid and sensitive method for the quantitation of microgram quantities of protein utilizing the principle of protein-dye binding. Anal Biochem 72, 248-254.

Carr, N. G. \& Mann, N. H. (1994). The oceanic cyanobacterial picoplankton. In The Molecular Biology of Cyanobacteria, pp. 27-48. Edited by D. A. Bryant. Dordrecht, Boston \& London: Kluwer.

Cathala, G. \& Brunel, C. (1975). Bovine kidney alkaline phosphatase: catalytic properties, subunit interactions in the catalytic process, and mechanism of $\mathrm{Mg}^{2+}$ stimulation. $J$ Biol Chem 250, 6046-6053.

Cheng, K. J. \& Costerton, J. N. (1973). Localization of alkaline phosphatase in three Gram-negative rumen bacteria. J Bacteriol 116, 424-440.

Doonan, B. B. \& Jensen, T. E. (1977). Ultrastructural localization of alkaline phosphatase in the blue-green bacterium Plectonema boryanum. J Bacteriol 132, 967-973.

Engels, D. H., Engels, A. \& Pistorius, E. K. (1992). Isolation and partial characterization of an L-amino acid oxidase and of photosystem II complexes from the cyanobacterium Synechococcus PCC7942. Z Naturforsch 47c, 859-866.

Garen, A. \& Levinthal, C. (1960). A fine structure genetic and chemical study of the enzyme alkaline phosphatase of E. coli. I. Purification and characterization of alkaline phosphatase. Biocbim Biophys Acta 38, 470-483.

Grossman, A. R., Schaefer, M. R., Chiang, G. G. \& Collier, J. L. (1994). The responses of cyanobacteria to environmental conditions: light and nutrients. In The Molecular Biology of Cyanobacteria, pp. 641-675. Edited by D. A. Bryant. Dordrecht, Boston \& London: Kluwer.

Healy, F. P. (1973). Characteristics of phosphorus deficiency in Anabaena. J Pbycol 9, 383-394.

Hulett, F. M., Bookstein, C. \& Jensen, K. (1990). Evidence for two structural genes for alkaline phosphatase in Bacillus subtilis. $J$ Bacteriol 172, 735-740.

Ihlenfeldt, M. J. A. \& Gibson, J. (1975). Phosphate utilization and alkaline phosphatase activity in Anacystis nidulans (Synecbococcus). Arch Microbiol 102, 23-28.

Kobori, H., Sullivan, C. W. \& Shizuya, H. (1984). Heat-labile alkaline phosphatase from antarctic bacteria: rapid $5^{\prime}$ end-labeling of nucleic acids. Proc Natl Acad Sci US A 81, 6691-6695.
McComb, R. B., Bowers, G. N. \& Posen, S. (1979). Structural features. In Alkaline Phosphatase, pp. 189-228. Edited by R. B. McComb, G. N. Bowers \& S. Posen. New York: Plenum Press.

Manoil, C. \& Beckwith, J. (1985). TnpboA : a transposon probe for protein export signals. Proc Natl Acad Sci US A 82, 8129-8133.

Messing, J., Crea, R. \& Seeburg, P. H. (1981). A system for shotgun DNA sequencing. Nucleic Acids Res 9, 309-321.

Michel, G. P. F. \& Baratti, J. C. (1989). Phosphate-irrepressible alkaline phosphatase of Zymomonas mobilis. J Gen Microbiol 135, 453-460.

Michel, G. P. F., Alvarez, E., Guzzo, J., Cami, B. \& Baratti, J. (1992). Cloning and expression in Escherichia coli of a phoA gene encoding a phosphate-irrepressible alkaline phosphatase of Zymomonas mobilis. FEMS Microbiol Lett 98, 103-108.

Miller, J. H. (1972). Experiments in Molecular Genetics. Cold Spring Harbor, NY: Cold Spring Harbor Laboratory.

Neu, H. C. \& Heppel, L. A. (1965). The release of enzymes from Escherichia coli by osmotic shock and during the formation of spheroplasts. J Biol Chem 240, 3685-3692.

Normand, P., Cournoyer, B., Simonet, P. \& Nazaret, S. (1992). Analysis of a ribosomal RNA operon in the actinomycete Frankia. Gene 111, 119-124.

Pearson, W. R. \& Lipman, D. J. (1988). Impoved tools for biological sequence comparison. Proc Natl Acad Sci US A 85, 2444-2448.

Ray, J. M., Bhaya, D., Block, M. A. \& Grossman, A. R. (1991). Isolation, transcription and inactivation of the gene for an atypical alkaline phosphatase of Synechococcus sp. strain PCC7942. J Bacteriol 173, 4297-4309.

Rippka, R. (1988). Isolation and purification of cyanobacteria. Methods Enzymol 167, 3-27.

Sambrook, J., Fritsch, E. F. \& Maniatis, T. (1989). Molecular Cloning: a Laboratory Manual, 2nd edn. Cold Spring Harbor, NY: Cold Spring Harbor Laboratory.

Staden, R. (1986). The current status and portability of our sequence handling software. Nucleic Acids Res 14, 217-232.

Von Tigerstrom, R. G. \& Stelmaschuk, S. (1986). Purification and characterization of the outer membrane associated alkaline phosphatase of Lysobacter enzymogenes. J Gen Microbiol 132, 1379-1387.

Wanner, B. L. (1987). Bacterial alkaline phosphatase gene regulation and the phosphate response in Escherichia coli. In Phosphate Metabolism and Cellular Regulation in Microorganisms, pp. 127-157. Edited by A. Torriani-Gorini, F. G. Rothman, S. Silver, A. Wright \& E. Yagil. Washington, DC: American Society for Microbiology. Wyckoff, H.W. (1987). Structure of Escherichia coli alkaline phosphatase determined by X-ray diffraction. In Pbosphate Metabolism and Cellular Regulation in Microorganisms, pp. 118-126. Edited by A. Torriani-Gorini, F. G. Rothman, S. Silver, A. Wright \& E. Yagil. Washington, DC: American Society for Microbiology.

Yanisch-Perron, C., Vieira, J. \& Messing, J. (1985). Improved M13 phage cloning vectors and host strains: nucleotide sequences of the M13mp18 and pUC19 vectors. Gene 33, 103-109.

Received 18 April 1995; revised 26 July 1995; accepted 10 August 1995. 